\title{
Rivalités et utopies impériales en Perse : les Britanniques et la « Karun River » au XIX siècle
}

Imperial Rivalries and the Search for Utopia in Persia: the British and the Karun River in the 19th Century.

Rivalitäten und imperiale Utopien in Persien : Die Briten und der „Karun River

“im 19. Jahrhundert

\section{Guillemette Crouzet}

\section{(2) OpenEdition}

\section{Journals}

Édition électronique

URL : http://journals.openedition.org/rh19/4571

DOI : $10.4000 /$ rh 19.4571

ISSN : $1777-5329$

Éditeur

La Société de 1848

\section{Édition imprimée}

Date de publication : 31 décembre 2013

Pagination : 133-152

ISSN : 1265-1354

\section{Référence électronique}

Guillemette Crouzet, "Rivalités et utopies impériales en Perse : les Britanniques et la «Karun River » au XIXe siècle », Revue d'histoire du XIXe siècle [En ligne], 47 | 2013, mis en ligne le 31 décembre 2016, consulté le 19 avril 2019. URL : http://journals.openedition.org/rh19/4571 ; DOI : 10.4000/rh19.4571 


\section{GUILLEMETTE CROUZET}

\section{Rivalités et utopies impériales en Perse: les Britanniques et la "Karun River» au XIX siècle}

Le golfe Arabo-Persique est fréquemment présenté par l'historiographie comme un espace "périphérique» aux XIX et $\mathrm{XX}^{\mathrm{e}}$ siècles. D'un point de vue politique tout d'abord, il n'aurait en effet constitué qu'une «marge» dans la géographie de l'Empire britannique. Sur le plan économique ensuite, il n'aurait été que très peu touché par le phénomène de «mondialisation " se déroulant à deux échelles, toutes deux "globales» : celle de l'Empire britannique d'une part et celle du monde d'autre part ${ }^{1}$. Cet article se propose de nuancer ces théories en étudiant le cas d'un fleuve peu connu, le Karun [cartes 1 et 2], qui fut au centre d'un vaste débat ${ }^{2}$ dans les années 1880-1890. Désigné comme "le seul fleuve navigable en Perse » , le Karun est l'objet d'un intérêt extrêmement vif entre 1870 et 1900, non seulement en Angleterre, mais également à une échelle macro-régionale, celle de l'espace maritime du Golfe, où sont présents la Russie, l'Allemagne et la Grande-Bretagne. Ainsi des surveys ${ }^{4}$ du Karun et de ses espaces riverains

1. Matthew S. Hopper, The African Presence in Arabia, Slavery, the World Economy and the African Diaspora in Eastern Arabia, 1840-1940, thèse de doctorat d'histoire, Université de Californie, 2006 : 'It has become conventional among historians of the Middle East to describe the nineteenth-century economic history of the region [il s'agit du Golfe] in terms of incorporation into a modern economic system in a peripheral role' (p. 117).

2. George N. Curzon, Persia and the Persian Question, Londres, Frank Cass, 2009 [1892], 2 volumes, et du même auteur, 'Leaves from a diary on the Karun river', Journal of the Society of Arts, May 10, 1889, volume 37, p. 479-715; 'The Karun river and the commercial geography of South-West Persia', Proceedings of the Royal Geographical Society, septembre 1890. Voir également John Gordon Lorimer, The Gazetteer of the Persian Gulf, Oman and Central Arabia, Cambridge, Cambridge Archives Editions, 2003; Mark Sever Bell Col., 'A visit to the Karun river and Kum', Blackwood's Edinburgh Magazine, avril 1889, volume CXLV, p. 454-490; Murdoch Smith, 'The Karun river as a trade route', Journal of the Society of Arts, 10 mai 1889, volume 37, p. 561-565; J. A. Saldanha, The Persian Gulf Precis, Selections from State Papers, Bombay, Regarding the East India Company's Connections with the Persian Gulf, with a Summary of Events, 1600-1800, Calcutta, 1908, 8 volumes; Henry Rawlinson, A Memoir of Major-general Sir Henry Creswicke Rawlinson, Londres, Longmans, 1898 et Henry Layard, Early Adventures in Persia, Susiana, and Babylonia, Londres, John Murray, 1894, William F. Ainsworth, The river Karun, an opening to British commerce, Londres, Allen \& Co, 1890, et Capitaine Samuel Cornell Plant, Middle East Archive Centre of Saint Anthony College, Oxford, cote GB165-0230.

3. George N. Curzon, 'The Karun river and the commercial geography of South-West Persia', loc. cit.

4. Conservé dans cet article, le terme de survey rend ici, derrière le mot anglais, la complexité de ces opérations, qui ne consistaient pas uniquement en des relevés topographiques. La richesse des informations collectées allait bien au-delà de ce que révèlent les cartes réalisées durant les expéditions. 
sont-ils conduits à partir des années 1870 . De plus, voyageurs et diplomates britanniques mentionnent à de nombreuses reprises à partir de 1870 ce fleuve et la province perse environnante, conçue comme une nouvelle "Mésopotamie», riche de mille potentialités. Surtout, Lord Curzon lui consacre plusieurs écrits et il a, en tant que principal avocat de cette entreprise, une place particulière dans les négociations avec le Shâh qui conduisirent à l'ouverture du fleuve à la navigation internationale en $1888^{5}$. La bataille pour obtenir auprès du Shâh des concessions afin d'exploiter le cours d'eau fut rude, notamment entre Anglais, Russes et Allemands ${ }^{6}$. L'épisode du Karun, entre 1870 et 1900, s'inscrit en conséquence dans un contexte fort particulier, celui du «Grand jeu» en Asie ${ }^{7}$, qui implique principalement la Grande-Bretagne et la Russie. Précisons qu'alors le spectre d'une poussée russe vers l'Empire des Indes est fortement présent dans les esprits. Les tensions sont vives entre les deux puissances du fait d'intenses rivalités politiques et économiques. Le Karun, dans les sources mentionnées, est donc conçu comme un «outil» spatial fondamental des impérialismes britannique et anglo-indien dans le Golfe. Il est à la fois un point d'appui économique permettant de résister à la présence commerciale russe dans le nord de la Perse ${ }^{8}$ et à la concurrence allemande qui s'accroît, mais également un axe stratégique pleinement intégré au dispositif de protection du Raj britannique9. Le projet d'ouvrir le Karun aux steamers britanniques doit également être compris dans le cadre de cette "diplomatie de la vapeur" pratiquée par les Britanniques dans de nombreux espaces de leur empire, et notamment dans la zone voisine de l'Iraq ottoman. Il s'agit d'un type d'impérialisme informel conduit par divers types d'acteurs et que l'on peut comparer aux politiques menées par les Ottomans ${ }^{10}$, les Russes ou les Allemands dans la zone du Golfe et de la Perse à la même époque.

5. John Gordon Lorimer, op. cit., volume 3.

6. Volker Langbehn, Mohammad Salama (eds.), German Colonialism : Race, the Holocaust, and Postwar Germany, New York, Columbia University Press, 2011; Ulrike Lindner, 'Imperialism and Globalization : Entanglements and Interactions between the British and German Colonial Empires in Africa before the First World War', German Historical Institute London Bulletin, 32, 2010, p. 4-28.

7. Edward Ingram, The beginning of the great Game in Asia, 1828-1834, Oxford, Clarendon Press, 1979 et In defence of British India: Great Britain in the Middle East, 1775-1842, Londres, Cass, 1984. Voir également Keith Neilson, Britain and the Last Tsar: British Policy and Russia, 1894-1917, Oxford, Oxford University Press, 1995; Fiona K. Tomaszewski A Great Russia: Russia and the Triple Entente, 1905-1914, Westport (Conn.), Praeger, 2002; Lorraine de Meaux, La Russie et la Tentation de l'Orient, Paris, Fayard, 2010; Svetlana Gorshenina, L'Asie centrale. L'invention des frontières et l'héritage russosoviétique, Paris, CNRS Éditions, 2012.

8. Vanessa Martin, Anglo-Iranian relations since 1800, Londres, Routledge, 2005 et The Qajar Pact: Bargaining, Protest and the State in Nineteenth-Century Persia, Londres, I. B. Tauris, 2005.

9. Robert J. Blyth, The Empire of the Raj, India, Eastern Africa and the Middle East, 1858-1947, New York, Palgrave Macmillan, 2003; James Onley, 'The Raj Reconsidered : British India's Informal Empire and Spheres of Influence in Asia and Africa, Asian Affairs, 40, 2009, p. 44-62, Thomas R Metcalf, Imperial Connections: India in the Indian Ocean Arena, 1860-1920, Londres, University of California Press, 2007.

10. Christopher Alan Bayly, 'Distorted Development: The Ottoman Empire and British India, circa 1780-1916', Comparative Studies of South Asia, Africa and the Middle East, volume 27, 2007, p. 332344. 
Par ailleurs, à une autre échelle, les projets concernant le Karun permettent de replacer le Golfe dans une perspective neuve, absente des travaux des historiens. L'ouverture de ce fleuve aux navires à vapeur modifie en effet considérablement le degré d'intégration régionale du golfe Arabo-persique. Par le Karun, celui-ci est connecté à plusieurs espaces que nous examinerons et sa place décisive, comme voie d'eau entre l'océan Indien et l'Asie centrale ainsi qu'entre l'Inde et la Méditerranée, sera considérée. L'étude du Karun témoigne par là même du phénomène de mondialisation du Golfe et de la Perse au XIX ${ }^{e}$ siècle, en voie d'intensification à partir de 1880. En Perse, les puissances européennes multiplient les pressions diplomatiques pour obtenir des concessions. Enfin, cette étude souhaite démontrer que les Britanniques développèrent dans ces années une véritable "pensée» de l'espace, forgée entre autres par le rêve de succéder aux grands empires présents dans la région depuis l'Antiquité et d'imprimer une marque durable sur les territoires. Avec le Karun et le rêve de créer autour du Golfe un espace de "prospérité» liant Orient et Occident, c'est bien le concept spatial de Moyen-Orient - avant que Valentine $\mathrm{Chirol}^{11}$ ne le nomme ainsi -, qui est en genèse dans ce projet britannique ${ }^{12}$.

$*$

\section{L'IMAGINAIRE D'UN ESPACE}

Pour étudier le débat entourant le Karun, il est important de s'intéresser en premier lieu aux descriptions du fleuve et de ses espaces proches. Les sources révèlent d'une part l'imaginaire qui est attaché à cette partie de la Perse $^{13}$, mais traduisent également la mise en place d'une rhétorique puissante de certains protagonistes britanniques qui veulent faire du Karun un axe économique et géostratégique d'importance régionale et mondiale.

\section{Un univers en déclin?}

Il serait trop long de revenir ici sur l'importance des surveys dans l'histoire de l'impérialisme britannique dans le Golfe. Les surveys ont véritablement contribué au processus de «mainmise» britannique sur cet espace à partir

11. Valentine Chirol, The Middle Eastern Question, or Some Political Problems of Indian Defence, New York, E.P. Dutton, 1903.

12. Cf., entre autres, Daniel Folliard, «La Terre Vague. L'évolution des représentations britanniques du Moyen-Orient, 1850-1914", thèse de doctorat d'histoire sous la direction de Fabrice Bensimon, Université Paris- IV Sorbonne, 2011.

13. Denis Wright, The English Amongst the Persians: Imperial Lives in Nineteenth-Century Iran, Londres, I.B. Tauris, 2001. 


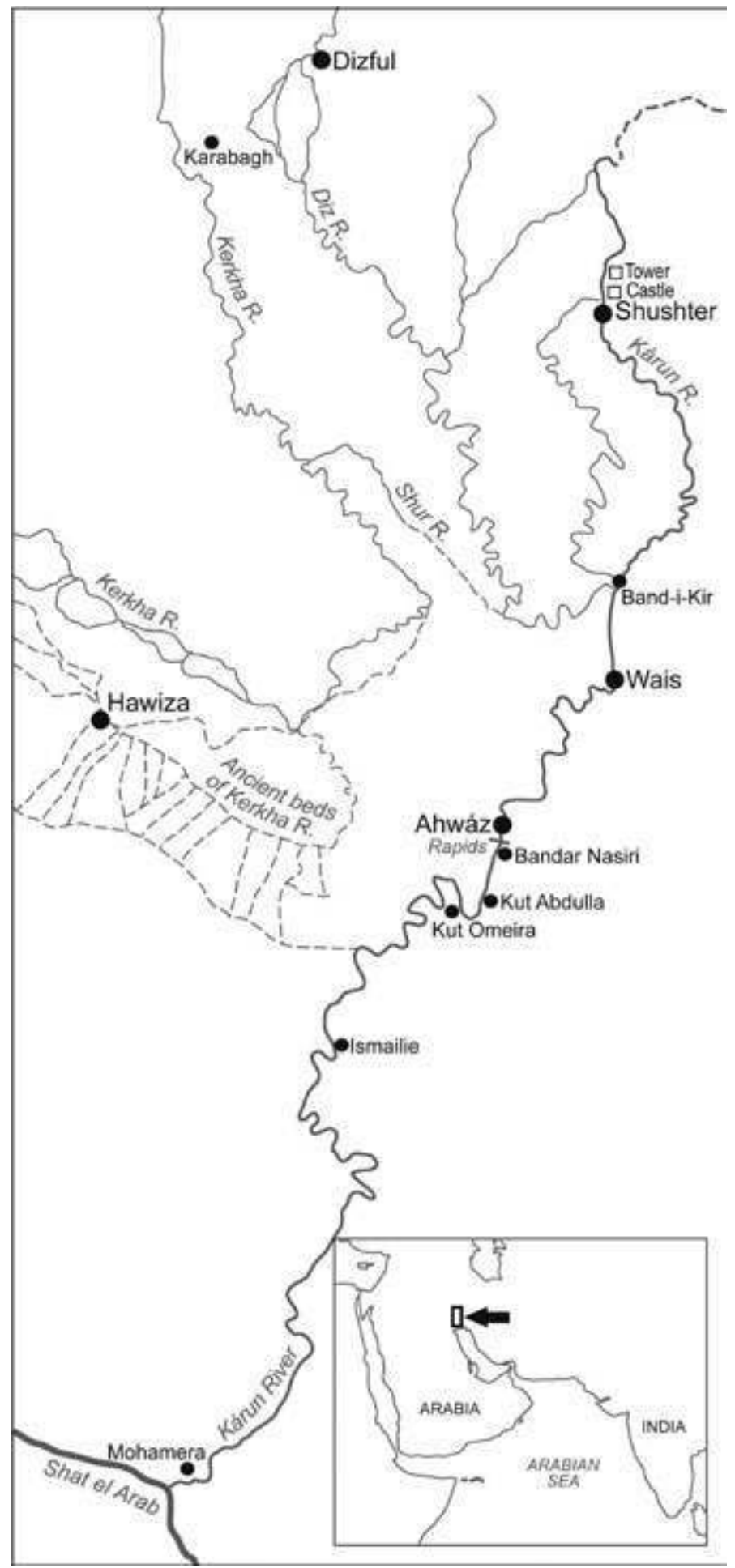

Carte 1. Le fleuve Karun au $\mathrm{XIX}^{\mathrm{e}}$ siècle 
des années $1810^{14}$. Il faut préciser que ces entreprises de vaste ampleur avaient des visées "politiques» certaines, auxquelles s'ajoutait une volonté de connaissance scientifique ${ }^{15}$. Dans la région qui nous occupe, un survey est conduit vers 1880 . Notons que dans les années 1830, tandis que des opérations de reconnaissance de grande ampleur étaient alors menées en Mésopotamie ${ }^{16}$, des hommes de l'entourage du Colonel Chesney avaient déjà remonté le fleuve ${ }^{17}$.

Une thématique principale domine l'ensemble des documents qui font référence au Karun : celle du déclin des espaces baignés par le fleuve. Il s'agit là bien évidemment d'un type de rhétorique fortement présent dans le discours impérial, visant à justifier présence et intervention ${ }^{18}$. Le même type d'arguments fut utilisé à la fin des années 1830, après le survey de Chesney, lorsqu'il fut question d'ouvrir le Tigre et l'Euphrate à la navigation à vapeur, puis dans le cadre d'autres projets britanniques en Mésopotamie ${ }^{19}$, tel que l'"Euphrates Railway Project».

Le colonel Sever Bell mentionne à la fin des années 1880 par exemple un paysage de canaux abandonnés ${ }^{20}$, au milieu de bâtiments ruinés. Les villes qui surplombent le cours du Karun sont aussi touchées par ce climat de désolation : il est question de villages et de cités en ruines ${ }^{21}$, à l'exemple de la "splendeur disparue ${ }^{22}$ d'Ahwaz. L'officier ne cache pas ses sentiments lorsqu'il visite Shustar : la saleté des rues le frappe ainsi que l'arrêt de la production de tapis qui plonge les populations dans la misère. Khoramabad connaît un sort assez similaire, elle aussi devenue une ville presque «fantôme». À ce tableau s'ajoutent d'autres remarques qui vont dans le même sens. La végétation, loin d'être luxuriante, frappe au contraire non seulement par son aspect "pauvre» et surtout "sauvage». Les palmiers dattiers sont malades en de multiples endroits : de petite taille et sans feuilles, ils ne produisent plus de fruits. Les marais sont omniprésents : les zones

14. Charles Rathbone, History of the Indian Navy, 1613-1863, 2 volumes, réed. Cambridge, Cambridge University Press, 1992, C.G. Constable, A.W. Stiffe, lieut., The Persian Gulf Pilot Including the Gulf of Oman, Londres, Hydrographic Office Publication, 1864.

15. Sur les surveys, voir entre autres, Kapil Raj, «La construction de l’empire de la géographie. L’odyssée des arpenteurs de Sa Très Gracieuse Majesté, la reine Victoria, en Asie Centrale», Annales, HSS, 1997, volume 52, n 5, p. 1153-1180; et Matthew Edney, Mapping an Empire. The Geographical Construction of India, Chicago, Chicago University Press, 1999.

16. Stuart A. Cohen, British Policy in Mesopotamia 1903-1914, Londres, Ithaca Press, 1976; John S. Guest, The Euphrates Expedition, Londres, Routledge, 1992.

17. Francis Rawdon Chesney, The Expedition for the Survey of the Rivers Euphrates and Tigris, Londres, Brown, Green, and Longman, 1850, volumes 1 et 2; Narrative of the Euphrates Expedition, Londres, Longmans, Green, and Co, 1868.

18. Cf. à titre de comparaison Diana K. Davis, Resurrecting the Granary of Rome: Environmental History and French Colonial Expansion in North Africa, Athens, Ohio University Press, 2007, et plus généralement, Richard Drayton, Nature's Government: Science, Imperial Britain, and the 'Improvement' of the World, Londres, Yale University Press, 2000.

19. Stuart A. Cohen, op. cit. et Halford L. Hoskins, British Routes to India, Londres, Frank Cass 1966. 20.'A visit to the Karun river and Kum', loc. cit., p. 465.

21. Ibidem, p. 465 et 481.

22. 'Vanished splendour', 'Leaves from a diary on the Karun river', loc. cit., p. 495. 
d'eau stagnantes ont remplacé, sous la plume des auteurs cités, les champs de céréales et les vergers. Lord Curzon évoque d'arides terrasses alluviales dépourvues de végétation ou encore laissées à l'abandon et gagnées par une flore "sauvage »" Les voyageurs reportent scrupuleusement dans leurs récits chaque arbre aperçu depuis les bateaux tant il semble qu'ils soient devenus rares dans ce paysage désertique et minéral. Les espaces riverains du Karun, jadis fortement anthropisés, sont désormais mortifères, à l'image de ces marécages qui se multiplient. L'homme a abandonné ce territoire pour céder la place à une faune d'animaux sauvages dont Lord Curzon relève la présence, évoquant les chacals, les sangliers et les lynx. Le gibier ne cesse de croître et les habitants, comme gagnés par une sorte de torpeur, ne se livrent plus à aucune activité cynégétique : sur les bords du Karun, Lord Curzon aperçoit à de nombreuses reprises des lièvres ainsi que des poules d'eau, des francolins ou encore des gangas. Une nature "sauvage» semble donc avoir repris ses droits dans le pays du Karun. En raison de l'absence d'activités économiques dynamiques, tant d'un point de vue agricole qu'industriel ou commercial, le déclin démographique des abords du Karun est particulièrement mis en avant par les Britanniques : les populations ont en effet migré vers d'autres régions de la Perse ${ }^{24}$. Lord Curzon, dans Persia and the Persian Question ${ }^{25}$, tente un recensement des populations du pays du Karun, citant ses sources et livrant des estimations variées. La conclusion est assez sévère : elle certifie un dépeuplement jugé préoccupant. Seules quelques tribus nomades qui vivent de leurs productions continuent d'évoluer de part et d'autre du fleuve. Elles vivent durant l'hiver dans de petites oasis, cultivant quelques fruits et légumes, produisant du fromage avec le lait de leurs bêtes et tissant la laine. Au printemps, elles se dirigent avec leur bétail vers les terrasses alluviales du fleuve où elles sèment puis récoltent des céréales qu'elles stockent ${ }^{26}$ pour la saison froide.

Aux thèmes de l'abandon et de la désertification du bassin hydrographique du Karun s'en ajoute un autre. En effet, les auteurs se disent d'autant plus surpris du spectacle qui s'offre à leurs yeux que le Karun s'inscrit, selon eux, dans l'espace géographique prospère de la Mésopotamie $^{27}$. Si l'extrême fertilité des rives voisines du Tigre et de l'Euphrate est sans doute quelque peu exagérée, elle permet d'accentuer la différence

23. 'A visit to the Karun river and Kum', loc. cit., p. 456; 'The Karun river and the commercial geography of South-West Persia, by the hon. G. Curzon', loc. cit., p. 10.

24. Ibidem, p. 19.

25. George N. Curzon, Persia and the Persian Question, op. cit.

26. 'The Karun river and the commercial geography of South-West Persia, by the Hon. G. Curzon', loc. cit., p. 9; 'A Visit to the Karun river and Kum', loc. cit., p. 457.

27. Guillemette Crouzet, "A Sea of Perpetual Maritime Truce?». Les Britanniques et le golfe Arabo-persique (vers 1800-vers 1914), thèse de doctorat d'histoire sous la direction d'Éric Bussière, Université Paris IVSorbonne, à venir, 2014. Cf. également Camille Lyans Cole, Hydraulics and the New Imperialism of Technology in Late Ottoman Iraq, Master of Philosophy in Historical Studies, Université de Cambridge, juin 2013. 


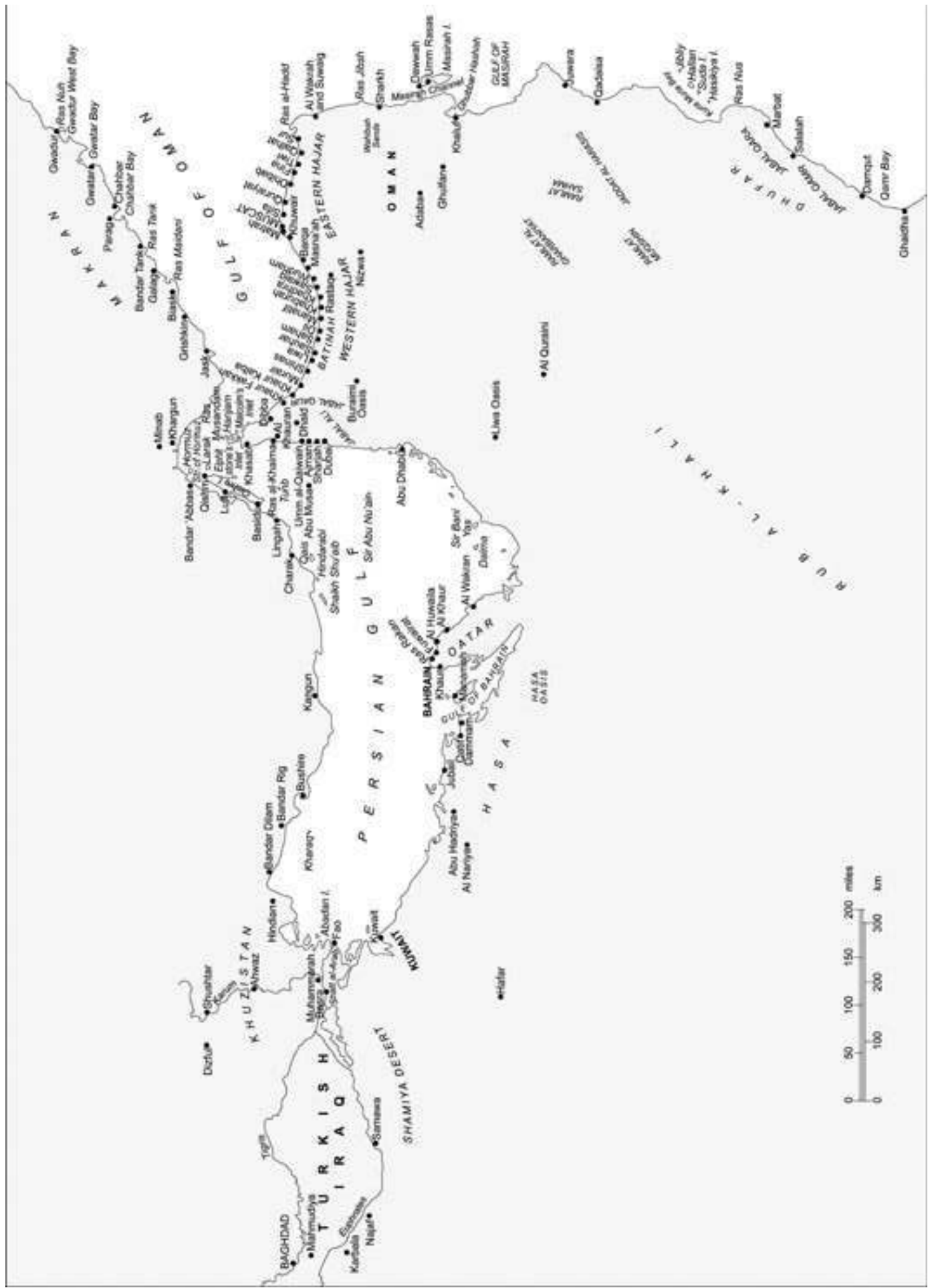


avec les abords du Karun décrits comme autant d'isolats de pauvreté dans un monde de richesses ${ }^{28}$. En outre, les descriptions des auteurs prennent sous leur plume un caractère «intemporel» et «mythique», relevant à la fois du temps biblique de la Genèse que de celui des légendes mésopotamiennes : décrivant les jardins d'Eden, un espace offrant mille potentialités où pourraient paître des troupeaux non loin de vergers regorgeant de fruits, surplombant des champs de céréales densément semés. Le fleuve Karun est alors une sorte "d'espace-temps" "mythifié" et le tableau qui en est fait n'est pas sans rappeler celui dressé par les auteurs qui mentionnaient le Shatt El Arab et la Mésopotamie dans les années 1830-185029. Si les sources soulignent que c'est non loin du Karun que se serait situé le Paradis $^{30}$, désormais la région appartient à un autre espace-temps, situé hors du monde de la Genèse, celui de la "chute», apportant son lot de ruines et de misères. Ces remarques jalonnent les écrits des arpenteurs britanniques qui, paradoxalement, lient rarement celles-ci à l'absence d'investissement des populations dans les travaux agricoles ${ }^{31}$. La critique est tout autre : c'est surtout l'inexistence de mesures prises par les Ottomans et par les Perses dans la région depuis des siècles que les auteurs dénoncent. Les Ottomans auraient en effet presque volontairement délaissé cette région de la Perse ${ }^{32}$ et auraient permis que toute forme d'activité économique s'éteigne ${ }^{33}$. Lord Curzon dénonce l'attitude des administrateurs de la Sublime Porte dans cette zone, qu'il qualifie d' "Ottoman impertinence " ${ }^{34}$. Des propos violents, teintés d'orientalisme ${ }^{35}$, soulignent «l'archaïsme" de ces gouvernements. L'ensemble des clichés propres à la littérature orientaliste sont présents dans les sources. Certains des textes mentionnés sont ainsi de véritables charges contre ceux qui sont désignés comme des "potentats orientaux" responsables du déclin observé. Malgré le caractère autoritaire de leur pouvoir, ils se définissent précisément par leur incurie et par l'absence de tout projet. Le Karun n'est donc qu'un reflet de la situation d'une Perse toute

28. Ibidem, p. 464-465.

29. William Ainsworth, Researches in Assyria Babylonia and Chadea, Forming Part of the Labours of the Euphrates expedition, Londres, J.W Parker, 1838; Francis Rawdon Chesney, The Expedition..., op. cit.; Lieut. H. B. Lynch, 'Note on a part of the river Tigris, between Baghdad and Samarrah', Journal of the Royal Geographical Society, volume IX, 1839.

30. Lord Curzon note que c'est près de Kurnah que se trouverait le site biblique du Jardin d'Eden. Kurnah, aujourd'hui appelée Qurnah, se situe en amont de Bassorah, sur le Tigre. $C f$. Charles W. J. Withers, 'Geography, Enlightenment, and the Paradise Question', in Charles W. J. Withers, David Livingstone (ed.), Geography and Enlightenment, Chicago, University of Chicago Press, 1999.

31. 'A visit to the Karun river and Kum', loc. cit, p. 475.

32. Le Karun est situé en territoire perse et n'est donc pas sous domination ottomane. Il est donc intéressant de noter que les auteurs anglais saisissent ici une sorte de prétexte pour bâtir un portrait-charge contre les Ottomans.

33. Ibidem, p. 455.

34. 'Leaves from a diary on the Karun river', loc. cit., p. 481.

35. Toby Dodge, «Stephen Hemsley Longrigg et ses contemporains : le despotisme oriental et les Britanniques en Irak (1914-1932)", Maghreb-Machrek, 2010, n² 204, p. 33-58. 
entière plongée, aux yeux des Britanniques, dans un déclin irrémédiable ${ }^{36}$. La condamnation britannique est d'autant plus forte que les espaces riverains du Karun sont dépeints comme riches de bien des promesses, ce qui renforce l'idée d'une sorte de "culpabilité" perso-ottomane. La mention de cette prospérité non exploitée autorise ainsi la mise en place d'une stratégie rhétorique favorable au projet britannique d'investissements de capitaux dans le bassin du Karun. Lord Curzon estime ainsi que seuls les Britanniques peuvent conduire cette région à retrouver une prospérité passée et pensée comme "naturelle», étant donné son emplacement géographique. Citant Ezéchiel, le colonel Bell pense qu'une forme d'interventionnisme économique mené par les Britanniques peut faire renaitre le Jardin d'Eden sur les rives du Karun ${ }^{37}$ et redonner vie à cet espace stratégique depuis l'Antiquité. Mysticisme et pragmatisme se mettent ainsi au service de l'Empire d'Albion.

\section{Le fleuve Karun : le mythe d'un âge d'or}

En effet, le processus de mythification rhétorique de la région et la justification de l'entreprise britannique sont complexes. Délaissés par les Ottomans et les Perses, en déclin alors que la zone devrait connaître la richesse en raison de sa localisation géographique, le Karun et ses espaces riverains sont également présentés comme un cœur économique et commercial, le centre "historique" des puissants empires qui se sont succédé depuis l'Antiquité. Une nouvelle fois, de nombreuses similitudes avec le discours britannique développé au sujet de la Mésopotamie tout au long du XIX ${ }^{\mathrm{e}}$ siècle doivent être soulignées. Ainsi, dans cette géographie imaginaire et historicisée, les grandes constructions politiques citées par les auteurs se sont-elles organisées au fil des millénaires autour du fleuve. Selon eux, les Empires babylonien, assyrien, mède, grec, parthe et sassanide auraient été bâtis autour d'un cours d'eau pensé comme un ombilic fondateur et presque "nourricier ${ }^{38}$. Il en va de même pour l'Empire romain qui a également imprimé sa marque dans l'espace, avec notamment les ruines d'un barrage construit sous le règne de Valérien. La domination politique du Karun semble ainsi avoir été un élément déterminant de la puissance et de la stabilité du monde depuis la période mésopotamienne. Dans une réflexion teintée d'un certain « romantisme», en contemplant des ruines sassanides, le major Murdoch note que tous les empires ont connu le déclin et ont disparu mais que le Karun est demeuré prospère jusqu'à une période récente en raison de sa position

36. Denis Wright, op. cit.

37. 'Leaves from a diary on the Karun river', loc. cit., p. 481.

38. On notera ici l'importance de l'influence de la pensée d'Edward Gibbon et de son ouvrage History of the Decline and Fall of the Roman Empire, Londres, John Murray, 1776-1789. 


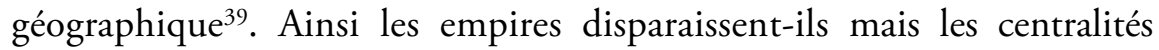
géographiques demeurent. Les critiques citées à l'égard de l'Empire ottoman prennent alors un sens différent : ce pouvoir est voué à l'échec car son cœur se trouve bien plus au nord de la zone évoquée, et non autour du Karun. $\mathrm{La}$ "légitimité» d'un empire ayant délaissé ce fleuve peut également être questionnée. À différentes échelles, le fleuve a en effet, d'après les sources, toujours représenté un centre, à tous les sens du terme : il a été le "point nodal» de divers empires et donc par excellence le "centre» du monde. D'un point de vue régional, ses rives ont toujours représenté des espaces attractifs vers lesquels marchandises et populations convergeaient ${ }^{40}$. Il est ici intéressant de noter qu'une représentation géographique quelque peu "floue» et mythifiée sert la rhétorique britannique : le Karun est ainsi constamment assimilé au Tigre et à l'Euphrate et au système fluvial de la Mésopotamie. À travers cette vision téléologique de l'histoire et dans cette représentation spatiale particulière du monde, le fleuve et le golfe Arabopersique sont donc des axes fondamentaux, à grande comme à petite échelle, structurant ces constructions économiques macro-régionales que sont les empires.

Ces considérations permettent par ailleurs de revenir sur les descriptions quelque peu «romancées» qui sont faites du fleuve. La diversité des paysages, la variété de la faune et de la flore mentionnées sont sans doute liées à une volonté de représenter le Karun comme un microcosme des mondes qu'il a dominés. En effet, dans un discours contradictoire, la végétation est peinte comme "pauvre" à l'image des palmiers dattiers malades, pour signifier le déclin, tout en étant étrangement variée. Les voyageurs évoquent des arbres appartenant aux mondes désertiques et "tropicaux", comme les palmiers ou des buissons épineux, mais aussi d'autres qui s'épanouissant dans des climats tropicaux plus humides comme les grenadiers. Certains des paysages bordant le Karun ressemblent, sous la plume des auteurs, à ceux que l'on peut associer à l'Europe du Nord, avec des forêts de feuillus et de conifères et des champs semés de blés. Des tourbières et des marécages sont également signalés. D’autres paysages sont plus «méditerranéens", avec des oliviers complantés d'arbres fruitiers, de rosiers parsemés d'espaces céréaliers, et une végétation de pins et de cyprès ${ }^{41}$. Des images renvoient ensuite aux représentations mentales qui sont liées aux zones désertiques : des petites "oasis» où s'épanouissent les palmiers qui surplombent les canaux d'irrigation forment des taches de verdure dans ces espaces minéraux. On peut compléter l'analyse en mentionnant à nouveau la faune. Comme la flore, celle-ci est extrêmement diverse et relève de celle que l'on associe aux différents espaces cités précédemment. Poules d'eau,

39. 'Leaves from a diary on the Karun river', loc. cit., p. 561.

40. Ibidem, p. 561.

41. 'A visit to the Karun river and Kum', loc. cit., p. 474. 
chèvres, bœufs, chevaux de trait, lézards côtoient des chacals, des chats sauvages et des chameaux. On pourrait alors avancer que le fleuve Karun serait en quelque sorte dépeint comme une "arche de Noé" où l'on trouve non seulement tous les animaux de la Création mais, aussi, un échantillon de la végétation de l'ensemble des espaces connus de l'homme. Elle est comme un conservatoire du monde ou plutôt des "mondes", et ce depuis l'Antiquité. Lorsque les auteurs peignent les habitants des bords du Karun labourant les champs et semant, les techniques ne semblent pas avoir évolué depuis la plus haute Antiquité. Le temps paraît s'être arrêté et le pays du Karun représente une sorte de "bulle» spatio-temporelle. Des bœufs, dans ce paysage comme figé dans le temps et dans l'espace, tirent lentement les charrues guidées par les paysans. De la même manière, le système d'irrigation des canaux semble inchangé depuis la période mésopotamienne. Peuton avancer que ces représentations sont à nouveau colorées d'orientalisme? Elles paraissent correspondre en effet à celles qui sont de façon traditionnelle attachées à l'Égypte et à la Mésopotamie et ont sans doute été reprises par les auteurs dans un souci de souscrire à des codes littéraires précis et de s'inscrire dans une certaine intertextualité. La représentation mentale attachée au Golfe, au Karun et à la Mésopotamie est donc complexe. D’une part, elle emprunte de nombreux traits à une certaine vision d'un Orient pensé comme "figé» et où évoluent des types de personnages bien particuliers comme le despote oriental, à l'image des Ottomans, ou des paysans égyptiens et mésopotamiens. La flore et la faune, avec les lézards et les chameaux, sont là pour satisfaire le goût des lecteurs pour l'exotisme. En un sens, sous ces plumes britanniques, le Karun et le Golfe correspondent à l'idée d'un Orient immuable que la Grande-Bretagne se doit de réveiller.

Cependant, l'histoire, précisément, semble avoir "récemment" repris son cours à un rythme lent. En effet, l'Empire britannique est pensé comme le successeur des différents empires qui l'ont précédé dans cette zone ${ }^{42}$. Il y a là un téléologisme fort des auteurs : d'une façon «logique», l'Empire britannique s'est installé dans cette zone prospère. À une vision particulière de l'espace s'ajoute chez les auteurs cités une "pensée» de l'histoire. L'histoire ne peut se faire et donc s'écrire qu'à travers celle des grandes formations impériales, selon un processus pensé comme "naturel» et irrémédiable. C'est ce que déclare le major Murdoch : "Notre empire a succédé à un autre ${ }^{43}$. Et les sources témoignent de la volonté des Britanniques de faire renaître le Karun à travers un projet précis et de renouer avec cette prospérité passée mythifiée. Vocation intemporelle et devenir du Karun sont donc ainsi liés. 


\section{L'ouverture DU KARUn À LA NAVIgation À VAPEUR}

\section{Aux origines du survey}

La nécessité d'accroître la somme d'informations disponibles au sujet du bassin du Karun vers 1870 et le soutien accordé au survey qui est conduit sont indissociables de la volonté britannique d'ouvrir ce fleuve à la navigation à vapeur. La bataille pour obtenir la concession du Karun fut rude. Diplomates, industriels et ingénieurs britanniques cherchèrent à bâtir le projet le plus précis et le mieux documenté afin de pouvoir rivaliser avec la compétition internationale.

Le survey, tout en livrant de nombreux détails, se concentre surtout sur des observations d'ordre hydrographique et hydrologique. Si le Karun est désigné, de façon récurrente, comme "la seule rivière (River, en anglais dans le texte) navigable en Perse», des obstacles se présentent pour que son cours soit remonté par des navires à vapeur. Le survey distingue six sections dont le caractère navigable n'est pas égal. Mark Sever Bell parle de "difficultés topographiques ${ }^{44}$ et d'un cours fort sinueux. Mais le principal problème réside dans la présence de dangereux rapides en aval d'Ahwaz : ceux-ci risquent en effet de créer des ruptures de charge et de rendre nécessaires des aménagements supplémentaires coûteux. En dépit de ces problèmes, le bilan du survey est plutôt positif. Les sources insistent en conséquence sur les bénéfices économiques qui seraient tirés de l'ouverture du fleuve. Ces derniers sont envisagés à différentes échelles : celle, d'une part, très «locale» du pays du Karun, mais aussi celle, macro-régionale, intégrant le Karun au système hydrographique du Shatt el Arab. Enfin, les contours d'une autre région se dessinent, dominée d'un point de vue politique et économique par les Britanniques, structurée par le Golfe et l'Inde et où le Karun est un indispensable micro-couloir de circulation des hommes et des marchandises. Une nouvelle fois, les sources traduisent la complexité de la vision de l'espace propre aux auteurs et, en particulier, à un personnage comme Lord Curzon. Le Karun est en effet conçu comme un axe important de pénétration des marchés, à une échelle micro-régionale comme macro-régionale tout en s'insérant dans le jeu impérial global de la Grande-Bretagne.

Considérons donc tout d'abord l'échelle la plus fine. En premier lieu, l'arrivée de capitaux étrangers permettrait de redynamiser le commerce et l'agriculture. Les va-et-vient des bateaux à vapeur sur le fleuve conduiraient les habitants à restaurer les canaux d'irrigation et à retourner vers les champs et les vergers. Sous la plume de Lord Curzon, les espaces agricoles riverains du Karun semblent s'éveiller lorsqu'il est question du projet britannique. Les navires pourraient acheminer vers le Golfe et l'Inde 
les produits agricoles dont les régions baignées par le Karun regorgent. Les biens commerciaux sont aussi pris en compte. Sont évoqués les villes et les villages qui bordent le fleuve et qui sont de petits centres de tissage. Les populations y travaillent la laine des moutons élevés par les tribus nomades, selon la tradition d'une complémentarité bien connue. À ce sujet, le colonel Sever Bell note le caractère rudimentaire des métiers à tisser. La production est faible et les tapis et couvertures confectionnés ne sont exportés que dans un espace extrêmement réduit. Selon le même analyste, l'existence d'une ligne régulière de navires de commerce convaincrait peut-être les populations d'investir dans le secteur textile et de le moderniser. Toutes les conditions seraient ainsi réunies pour que l'artisanat textile local connaisse une expansion. Le futur vice-roi des Indes souligne que le principal bénéfice de l'ouverture du Karun pour les habitants de ce pays serait un meilleur approvisionnement des marchés et des zones enclavées, atteignables jusqu'alors uniquement via des routes caravanières. Les produits phares exportés sont égrenés au fil de la plume : «blé, laine, coton, opium, fruits secs, peausseries, beurre, tabac, huiles végétales, auxquels s'ajoutent des tapis, des couvertures et même peut-être du vin ${ }^{45}$. Par des effets d'entraînement, dynamisme, commerce en développement et prospérité deviendraient rapidement, selon Curzon, la règle dans les espaces riverains du Karun. L'ouverture des marchés et l'augmentation des importations et des exportations seraient pour Sever Bell la clef d'un possible avenir économique : «Prenons en considération l'importance du Karun comme un moyen de développer les importations et les exportations du commerce étranger en Perse ${ }^{46}$. C'est bien véritablement à une sorte d'espace économique micro-régional structuré par le Karun que les Anglais souhaitent donner naissance avec leur projet. Le terme de "trade route", employé par le major Murdoch, prend ici tout son sens. Les effets du projet sur la démographie sont par ailleurs longuement analysés dans les sources. La naissance d'une micro-industrie textile dynamique comme la reprise de la production agricole entraîneraient le retour des habitants ayant délaissé les rives du fleuve et la renaissance régionale.

Les bénéfices économiques présumés sont également présentés à une autre échelle. En effet, aux dires de Lord Curzon, le Karun est une zone géostratégique essentielle dans un processus permettant de pénétrer au cœur de la Perse et de l'Asie centrale, dans des territoires dont l'extrême fertilité est sans cesse rappelée. L'ouverture du fleuve permettrait ainsi d'atteindre d'une façon "sûre ${ }^{47}$ des territoires convoités pour leurs richesses.

45. 'The Karun river as a trade route', loc. cit., p. 564.

46. 'Importance of the Karun as a means of developing the export and import foreign trade of Persia', Ibidem.

47. De nombreux documents soulignent le caractère dangereux des routes caravanières de l'intérieur de la Perse. J. M. Dening (ed.), Political diaries of the Persian Gulf, 20 volumes, Cambridge, Cambridge 
Les espaces riverains du Karun sont pauvres mais leur degré de connectivité à des cités dont la richesse rappelle presque celle des Mille et Une Nuits est fort et ancré historiquement. Les auteurs et les conclusions du survey rappellent sans cesse l'existence de traditions d'échanges économiques qui lient depuis des millénaires la Perse, l'Asie Centrale, la Mésopotamie, l'Inde, le Golfe et la péninsule arabique. La trame des routes caravanières est inscrite dans l'espace-temps analysé, et redonner vie à ce tissu commercial apparait alors comme presque naturel. On peut avancer sans risque que les perspectives de développement à une échelle micro-régionale, celle du pays du fleuve Karun, n'intéressent que modérément les Britanniques. Leur but véritable est de se positionner sur les marchés de l'intérieur de la Perse, de l'Irak et de l'Asie Centrale, à une échelle macro-régionale. Le futur viceroi des Indes évoque ainsi les «les grandes villes et les grands centres de la culture des céréales de l'Ouest de la Perse ${ }^{48}$. Le coton, le blé, l’opium, le riz et le tabac y pousseraient en quantité ${ }^{49}$. Mark Sever Bell mentionne le Loristan et les villes de Khoramabad et de Borujird : on y remarquerait des vignes splendides, dont les raisins seraient d'une taille extraordinaire. Concernant Hamadan, la qualité d'un vin produit par des Arméniens est vantée tandis que sont mis en avant les souhaits de le commercialiser dans la région. Par ailleurs, Hamadan qui est aussi spécialisée dans le séchage des fruits pourrait voir exporter ses productions.

À lire les Britanniques qui passent dans la région, il semble que tous les types de produits (bois, céréales, fruits et légumes) soient disponibles et que leur récolte ou leur production puisse en être augmentée comme l'avance Curzon. Des denrées rares pourraient également bénéficier de la présence d'une ligne de bateaux à vapeur et être ainsi exportées : la région est en effet caractérisée par des plantes médicinales fort réputées ${ }^{50}$. Les plantes tinctoriales de prix sont une caractéristique de la région de Dizful et de Shushter et Lord Curzon souhaite voir leur culture s'intensifier, sans doute à destination du marché anglo-indien. Les richesses minières sont aussi mentionnées et notamment celles de Kum et ses mines de cobalt. Puis, ce sont surtout des villes disposant d'une industrie de tissage de la laine et du coton atteignant des prix élevés ${ }^{51}$, qui sont longuement prises en compte. Il en va ainsi de Sultanabad et ses tapis d'une grande beauté ${ }^{2}$ tout comme certains centres de l'Irak ottoman et du Kurdistan ${ }^{53}$. Malgré leur splendeur, les tapis ne sont que peu exportés et atteignent des prix extrêmement élevés en

Archive Editions, 1990 et A. L. P. Burdett, A. Seay (ed.), Iran in the Persian Gulf, 6 volumes, Cambridge, Cambridge Archive Editions, 2000.

48. 'Great cities and centres of grain cultivation in the West of Persia', 'The Karun river and the commercial geography of South-West Persia', op.cit., p. 9.

49. Ibidem.

50. Ibidem, p. 18.

51. Ibidem.

52. 'The Karun river as a trade route', loc. cit., p. 565.

53. Ibidem., p. 564. 
raison de l'absence de moyens de transport rapides connectés aux grandes voies de commerce. Lord Curzon appelle de ses vœux la modernisation technique de ces centres de tissage : toutes les conditions seraient alors réunies pour faire des tapis la principale denrée exportable à une échelle macro-régionale. La ligne de steamers, les champs fertiles de coton de l'intérieur de la Perse ainsi que les zones de pâturages où paissent les moutons soutiendraient en continu cette expansion ${ }^{54}$. Dans la même veine, un futur similaire est envisagé pour les soieries d'Isfahan.

Intérêts commerciaux et politiques sont donc étroitement imbriqués dans cet espace ${ }^{55}$. Le Karun est un outil spatial pour accroître la puissance économique et politique britannique face aux impérialismes russe et ottoman. Le projet britannique repose par ailleurs sur la possible intégration du Karun au dispositif de protection de l'Empire des Indes et à son verrouillage ${ }^{56}$.

\section{Le Karun : un axe anglo-indien}

En premier lieu, le Karun est perçu, de façon multiscalaire, comme une voie stratégique permettant la pénétration des produits anglo-indiens en Perse. Une nouvelle fois, l'échelle micro-régionale du pays du Karun est évoquée. Mais la région est aussi, à une échelle plus fine, un axe géopolitique majeur dans le grand jeu qui oppose Britanniques et Russes en Asie centrale. En outre, il se révèle être, économiquement un point névralgique facilitant le développement de nouveaux marchés qui peuvent s'articuler à diverses échelles. Le Karun n'est, en un sens, que l'axe autour duquel les auteurs cités veulent organiser ce mouvement de conquête économique et commerciale vers le nord de la Perse. Comme le note Curzon, vers 1880, le sud de la Perse est pleinement dominé par les Britanniques ${ }^{57}$. Il s'agit là d'un acquis. Les produits anglo-indiens sont omniprésents dans cette région qui est connectée, via les steamers de compagnies britanniques ou indiennes naviguant dans le Golfe, à Bombay et au sud-ouest de l'Inde. Le sud de la Perse se situe pleinement dans cette zone d'influence anglo-indienne composée du nord de l'océan Indien, du Golfe et de l'Inde. Cependant, le nord reste à dominer et il est ici intéressant d'analyser la stratégie rhétorique qui est celle des Britanniques. Leur implantation économique en Perse septentrionale est, en effet, décrite comme un «fardeau impérial. Il s'agit d'une entreprise longue et coûteuse, volontiers présentée comme désintéressée. Les Britanniques contribueraient au «réveil " économique et commercial de toute la Perse et permettraient au Shâh et aux populations de renouer

56. James Onley, «L'empire britannique informel dans le Golfe (1820-1971)", Maghreb-Machrek, volume 187, 2006, p. 101-113.

57. Denis Wright, op. cit. 
avec leur passé glorieux. Selon les sources, la Perse aurait, comme le montre son histoire, un rôle à jouer dans l'économie régionale et mondiale et les Britanniques pourraient l'y aider ${ }^{58}$. Une Perse puissante, alliée des Britanniques et dominant l'Orient, serait donc un atout de taille pour les Britanniques dans cette région du monde.

On retrouve là une constante de la politique anglo-indienne dans le Golfe : les Britanniques veulent maîtriser l'espace tout en intégrant pleinement à leurs plans les puissances régionales. C'est une sorte de concert anglo-oriental, conduit par la Perse et la Grande-Bretagne, qu'ils appellent de leurs vœux ${ }^{59}$. À l'opposé, l'impérialisme russe est présenté comme prédateur et conduisant à l'asphyxie de l'économie persane : les Russes sont accusés d'avoir fermé aux Européens les marchés du nord d'un pays qu'ils conçoivent comme une chasse gardée monopolistique pour leurs produits ${ }^{60}$. Les Britanniques proposent bien évidemment, à l'opposé, d'ouvrir cette portion du territoire perse au commerce et aux investissements étrangers : les bénéfices du libéralisme et de la libre-concurrence sont longuement cités $^{61}$. De plus, la mission britannique revêt parfois un caractère civilisateur, en sus des progrès économiques apportés. Grâce aux mesures prises sous l'impulsion britannique, l'ordre succèdera à l'anarchie, les populations nomades se sédentariseront et la prospérité remplacera la pauvretét ${ }^{2}$. La Perse atteindrait alors une sorte d'état idéal, dont les descriptions ne sont pas sans rappeler celles d'un Karun "Jardin d'Eden". Il s'agit presque de conduire les Perses vers un stade de la "civilisation" qu'ils ne connaissent pas encore. Les Russes, par leur présence «asphyxiante», constituent donc un obstacle sur ce long chemin.

Résumons ces projets. Le système permettant l'avancée britannique au nord de la Perse est complexe. Le fleuve Karun est ainsi placé au centre d'un large dispositif de moyens de communication se déployant au nord, au sud ainsi qu'à l'ouest et à l'est. Le projet britannique, tel qu'il est présenté dans les sources, comporte de nombreux points qui sont flous. Le coût des investissements n'est jamais cité et la géographie de la trame ferroviaire manque grandement de précision. Néanmoins, le premier objectif des Britanniques est de développer les liaisons entre les différents moyens de communication, terrestres, fluviaux et maritime, tout en modernisant ces derniers et leurs structures. L'utopie britannique repose surtout sur la naissance d'une macro-région économique structurée par le Karun, le Shatt el Arab et le Golfe, et en relation avec d'autres mondes, comme nous allons le voir. On notera qu’à cette fin les Britanniques désirent pleinement associer les

58. Mark Sever Bell, 'A visit to the Karun river and Kum', loc. cit., p. 480.

59. Guillemette Crouzet, "A Sea of Blood and Plunder', Lutte contre la traite et politique impériale britannique", Monde(s), Espaces, Relations et Sociétés, n 1, mai 2012, p. 213-235.

60. 'The Karun river as a trade route', loc. cit., p. 561.

61. 'A visit to the Karun river and Kum', loc. cit., p. 480.

62. Ibidem, p. 481. 
Perses à leur entreprise. La coopération des milieux économiques est désirée et surtout le soutien et l'investissement du Shâh apparaissent comme indispensables. Il est notamment souligné que la mise en place d'une active politique persane de renforcement de la sécurité des routes est une composante essentielle du projet britannique.

Tout d'abord, une première vague d'investissements concernerait les voies fluviales. Les auteurs souhaitent que soit restauré un canal qui connecte le Karun au détroit du Shatt el Arab : le Haffar Canal qui constitue la voie d'eau majeure d'un réseau structurant l'Est de la Perse et la zone mésopotamienne. Il s'agit surtout de mettre en réseau les voies fluviales, avec le Karun et le Shatt el Arab, et la voie maritime, à travers le Golfe. Une connexion rapide entre le Golfe et ces fleuves faciliterait ainsi les liaisons économiques entre l'Inde, l'océan Indien, la Perse et l'Empire ottoman. La définition du Golfe comme un couloir maritime stratégique, entre terres et mers prend donc ici tout son sens. En second lieu, un plan de construction de voies ferrées et routières, reliées au Karun, représente la deuxième priorité des plans britanniques dans la région. Les auteurs désirent développer les échanges économiques entre plusieurs microrégions de la Perse, prospères, et créer un vaste espace commercial de dimension macro-régionale, où un train moderne permettrait aux hommes et aux marchandises de circuler plus aisément. L'objectif britannique, par ces routes et ces trains, est d'unifier l'espace et de lutter contre sa fragmentation en micro-espaces économiques. En effet, toujours selon Lord Curzon, l'économie perse reposerait sur des axes précis et des routes caravanières ancestrales. Kerman et le Sud-Est de la Perse exportent leurs produits via le port de Bunder Abbas. Fars et Shiraz seraient tournées vers Bushire tandis que des cités plus septentrionales, comme Ghilan, Mazanderan, Asterabad et une partie de la région du Khorasan, regarderaient vers les ports de la mer Caspienne et subiraient donc fortement ce qui est désigné comme une "tyrannie économique» russe. L'Azerbaidjan et sa capitale Tabriz ainsi qu'Erzérum et Trébizonde relèveraient de la structure du réseau des routes du Caucase. Enfin, une ultime microrégion est mentionnée, celle de Kermanshah qui appartiendrait au système du Tigre, de l'Euphrate et du Shatt el Arab. La fragmentation de ce tissu économique où les relations entre voie de terre et de mer sont inexistantes expliquerait, une nouvelle fois, l'absence de dynamisme de l'économie perse. Une ligne principale s'appuyant sur ce que les auteurs désignent comme "l'artère commerciale» de la Perse représente le cœur du dispositif. Les trains circuleraient du nord au sud entre Téhéran, Ispahan, Kashan, Koom, Hamadan, Sultanabad, Dowletabad, Burujird, Shushter et Mohammerah, donc de la capitale perse considérée comme le nœud des routes caravanières ancestrales sillonnant l'Asie Centrale et le Caucase aux rives septentrionales du Golfe. 
Le projet britannique a une dimension encore plus vaste. En effet, c'est presque d'un système des "trois mers» et des "trois mondes» dont les Britanniques rêvent. Tout d'abord, Méditerranée, golfe Persique, océan Indien et mer Caspienne seraient mis en réseau via le Shatt el Arab, le Karun et les voies ferroviaires, routières et caravanières. Ensuite, Mésopotamie, Asie Centrale et péninsule Arabique rentreraient en communication à une vitesse nouvelle. Dans l'imaginaire de la succession des empires, les Britanniques imprimeraient sur l'espace une marque particulière en bâtissant précisément un nouvel espace-temps. La dimension politique de ces rêves britanniques s'explique alors de façon claire. En effet, le problème de la puissance politique russe au nord de la Perse constitue une véritable obsession à leurs yeux. La Perse n'est qu'une périphérie spatiale de l'espace conquis par les tsars au fil des siècles en Asie Centrale, mais la menace est jugée comme réelle. En tissant des relations économiques durables, les Russes se sont implantés politiquement en Perse, comme le notent les auteurs. Selon les sources, c'est par leur présence économique forte que les Russes maintiennent leur suprématie dans cette zone : la politique de séduction conduite par les diplomates des Romanov à l'égard de Téhéran est notamment citée à plusieurs reprises. Le major Murdoch mentionne quant à lui les produits russes exportés vers Téhéran et sa région via les ports de la mer Caspienne et les réceptions offertes par l'ambassadeur russe à Téhéran aux milieux marchands et intellectuels de la capitale perse ${ }^{63}$. Pour les Britanniques, cette présence au nord de la Perse représente en effet un double danger : tout d'abord, la zone constitue un ourlet stratégique donnant accès aux mers et permettant ainsi d'atteindre le lac britannique, le golfe Arabo-persique, et donc par là même l'océan Indien et les Indes. D'autre part, les positions russes en Perse septentrionale pourraient donner accès, via les plaines d'Asie Centrale et les cols himalayens, au nord de l'Empire des Indes. Le spectre imaginaire d'une possible invasion russe en Inde est en effet extrêmement vif dans les esprits dans les décennies 1870 et $1880^{64}$. En pénétrant en territoire perse par le Karun et en menant à bien leurs entreprises, les Britanniques concurrenceraient de façon forte les Russes d'un point de vue politique. Enfin, on peut également considérer que le Karun pourrait représenter un axe stratégique dans l'hypothèse où un conflit armé éclaterait entre Russes et Britanniques. Par ce fleuve, des troupes pourraient aisément être acheminées vers le nord de la Perse et la Caspienne, et intervenir en cas de menace sur la frontière occidentale et septentrionale de l'Empire des Indes.

Enfin, le projet britannique est une arme de guerre contre une autre puissance impériale : l'Empire ottoman. En effet, à partir de 1870, le Golfe

63. 'The Karun river as a trade Route', loc. cit., p. 565.

64. Arnold Keppel, Gun Running and the Indian North-West Frontier, Londres, John Murray, 1911. 
est l'objet d'un regain d'intérêt de la part du pouvoir d'Istanbul. Entre autres démonstrations de force, un fort fut bâti à Fao à partir de 1887, soit à l'embouchure du Shatt el Arab. Cette initiative représentait en outre une violation du traité d'Erzeroum et démontrait surtout la volonté des Ottomans de faire du Shatt el Arab et du nord du Golfe une zone économique ottomane, tandis qu'une vaste politique de réformes était menée dans les provinces arabes de cet Empire ${ }^{65}$, alors peut-être appelé à tort "l'homme malade de l'Europe». Le projet britannique représentait donc un moyen de gagner en puissance dans un espace convoité et de renforcer "le lac britannique».

En 1888, après une bataille diplomatique âpre, le Shâh de Perse ouvrit le Karun à la navigation internationale. Une concession pour la construction de relais de charbon et autres infrastructures nécessaires aux steamers fut donnée par le Shâh à la firme britannique Lynch $^{66}$. Cette société avait déjà été chargée de veiller sur la ligne remontant le Shatt el Arab et, en 1888, le premier bateau qui remonta le Karun appartenait à la flottille des steamers mésopotamiens ${ }^{67}$. Vers 1901, Lord Curzon évoque deux ou trois vapeurs naviguant sur le Karun jusqu'à Ahwaz. Mais les sources manquent totalement de détails précis concernant les bénéfices économiques du projet. Il est donc impossible d'avancer quoi que ce soit sur l'aboutissement du rêve britannique de voir s'épanouir en Perse des espaces micro et macrorégionaux profondément connectés entre eux, où les produits britanniques auraient dominé les marchés, pas plus qu'il n'est possible de s'étendre sur la constitution d'un système des "trois" mers. En outre, il semble que graduellement, après 1908 et avec la relative accalmie des tensions dans le cadre de la fin du Great Game of Asia, le Karun ait été jugé comme un axe moins stratégique. Après 1918, avec l'effondrement de l'Empire ottoman, la création du mandat britannique en Iraq notamment et les débuts de l'aventure pétrolière, les préoccupations au sujet du Karun furent de moins en moins vives. Ces projets britanniques démontrent néanmoins - et c'est là sans doute leur principal intérêt - l'existence d'une véritable

65. Frederick F. Anscombe, The Ottoman Gulf: The Creation of Kuwait, Saudi Arabia, and Qatar, New York, Columbia University Press, 1997; Selim Deringil, 'They Live in a State of Nomadism and Savagery : the Late Ottoman Empire and the Post Colonial Debate', Comparative Studies in Society and History, volume 45, $\mathrm{n}^{\circ}$ 2, avril 2003, p. 311-342; et The Well-Protected Domains: Ideology and the Legitimation of Power in the Ottoman Empire 1876-1909, New York, I.B Tauris, 1999.

66. Stephanie Jones, 'The Management of British India Steamers in the Gulf 1862-1945 : Gray Mackenzie and the Mesopotamia Persia Corporation', in Richard Lawless (ed.), The Gulf in the Early 20th Century : Foreign Institutions and Local Responses, Durham, University of Durham Occasional Papers Series, 1986.

67. John P. Parry, 'Steam Power and British Influence in Baghdad, 1820-1860', The Historical Journal, volume 56, 2013, p. 145-173. 
vision britannique de l'espace et de l'importance stratégique du Golfe, comme voie maritime d'importance macro-régionale et mondiale. Ainsi, l'histoire de la concession du fleuve Karun témoigne-t-elle de la genèse, bien avant Valentine Chirol, du concept de Moyen-Orient, au sein duquel le Golfe occupe une place centrale et structurante. C'est d'un monde liant Orient et Occident, connectant mers et océans, terres et continents, dont les Britanniques avaient rêvé, mais surtout d'un univers à jamais en paix et prospère, car vivant sous une domination pensée comme perpétuelle. Autour du Karun et du Golfe, c'est bien une utopie spatio-temporelle qui se cristallisa tout au long du XIX ${ }^{\mathrm{e}}$ siècle et qui fut comme révélée à travers ce projet et la pensée de Lord Curzon. La vision de l'histoire que traduisent ces textes est également intéressante : ne peut-on pas avancer qu'à travers cet exemple, les Britanniques concevaient leur empire comme une sorte de stade ultime de l'histoire, seul capable de renouer avec l'espace-temps biblique?

Il demeure par ailleurs que le Karun continua longtemps de faire rêver les voyageurs et notamment Thomas Edward Lawrence ("Lawrence d'Arabie») qui le remonta en 1916. Ce dernier, tel ses prédécesseurs, s'étonne dans son récit de la pauvreté du pays et de l'absence d'habitants cultivant le sol. Mais il s'émerveille du spectacle d'une végétation luxuriante peuplée d'oiseaux aussi rares que merveilleux, perdus dans cet espace hors du monde et presque proche de l'état "idéal " du temps de la Genèse et du Paradis perpétuel.

Guillemette Crouzet est doctorante en histoire contemporaine et ATER à l'Université de Paris-IV Sorbonne (UMR IRICE 8138) 